\title{
Silicon-containing particles in synovial fluid: scanning electron microscopy coupled with analytical techniques allows an easy identification and differentiation from pathologically relevant crystals
}

\author{
GILBERT FAURE, ${ }^{12}$ PATRICK NETTER, ${ }^{13}$ AND MARIE-CHRISTINE BENE \\ From the ${ }^{1}$ Clinique Rhumatologique, the ${ }^{2}$ Laboratoire d'Immunologie, and the ${ }^{3}$ Laboratoire de \\ Pharmacologie, Centre Hospitalier et Universitaire de Nancy-Brabois, France
}

SUMMARY Silicon-containing particles were observed by scanning electron microscopy (SEM) in synovial fluid samples from patients with crystal-induced or inflammatory synovitis, or both. This material was an artefact produced by the technical procedures, but these particles could be easily differentiated from naturally occurring compounds by their morphology and their composition determined by analytical spectrometry.

Key words: synovial fluid, scanning electron microscopy, crystals, silicon, $x$-ray spectrometry.

Among the different techniques available to demonstrate the presence of microcrystalline compounds in synovial fluid and articular tissue scanning electron microscopy is reliable and allows the study of relatively large samples. When coupled with analytical devices, SEM provides, in a short time, a large amount of information about the composition of a sample. ${ }^{1-3}$.

Two recent papers have dealt with the identification of silicon-containing particles in synovial fluid. One study relates these particles to an occupational disorder. The other emphasises the artefactual nature of such material observed by transmission electron microscopy. ${ }^{5}$ In view of our experience with scanning electron microscopy we can confirm this latter possibility.

\section{Materials and methods}

Between 1977 and 1981 synovial fluid was obtained from 50 subjects suffering from various rheumatological disorders. All samples were aspirated under sterile conditions, with latex gloves and through

Accepted for publication 1 October 1984.

Correspondence to Dr G Faure, Laboratoire d'Immunologie, Faculté A de Médecine, BP 184, 54505 Vandoeuvre les Nancy Cedex, France. disposable plastic syringes. They were then collected in sterile vials without anticoagulant or glass balls. ${ }^{3}$

Subsequently, two methods were used to concentrate the constituents of the synovial fluid and to study their cellular and microcrystalline elements. In the first technique $10 \mathrm{ml}$ of synovial fluid was mixed with $125 \mathrm{IU}$ of testicular hyaluronidase and incubated for $15 \mathrm{~min}$ at $20^{\circ} \mathrm{C}$. After $20 \mathrm{~min}$ centrifugation at $5000 \mathrm{~g}$, the supernatant was discarded and 5 $\mathrm{ml}$ of a trypsin solution (125 $000 \mathrm{IU})$ was added to the pellet. The samples were further incubated for another 4 hours, centrifuged, and the new pellet washed with absolute ethanol and air dried. The residue obtained was finally placed on an amorphous adhesive holder and metallised with gold.

In the second method synovial fluid was merely held in suspension by a buffered fixative $(2 \%$ o glutaraldehyde and $2 \%$ paraformaldehyde in $10 \mathrm{mM}$ cacodylate buffer, $\mathrm{pH} 7 \cdot 3$ ). The whole mixture was $\mathrm{N}$ then sieved through a Millipore filter $(0.22 \mu \mathrm{m}$ pores) which was then placed in buffer, dehydrated in graded ethanol baths, and dried at critical point. The filter was finally glued to a holder and metal- $\mathscr{\Phi}$ lised with gold.

Scanning electron microscopy was performed with either a Cameca MEB 07 microscope equipped with $\mathbb{D}$ a wavelength dispersive $x$-ray spectrometer, or a $\frac{\rho}{\mathbb{D}}$ Cambridge Stereoscan 250 microscope coupled to an electron dispersive $x$-ray spectrometer. 


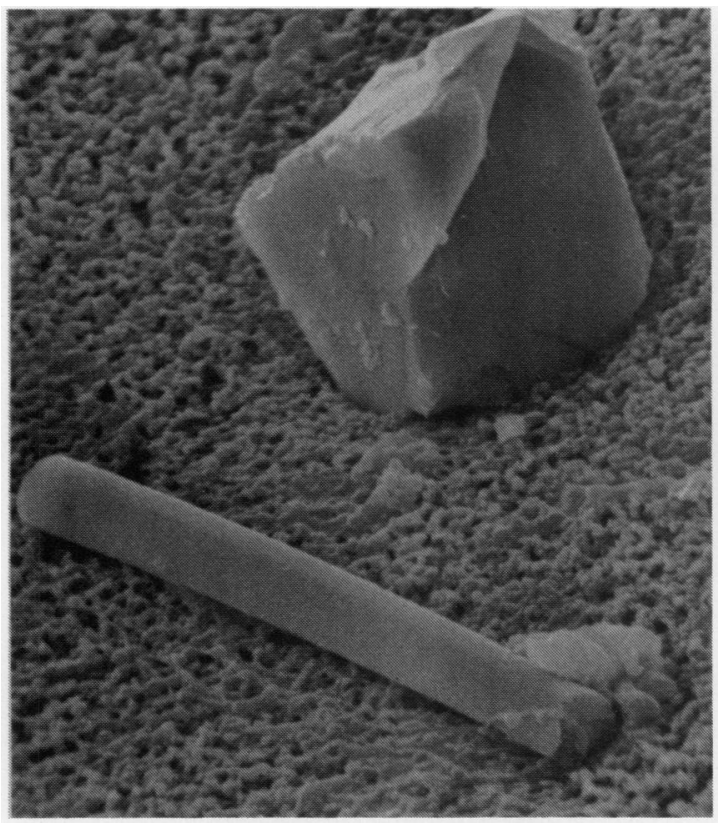

Fig. 1 Two silicon-containing particles collected on a Millipore filter from human articular synovial fluid. (× 4000).

\section{Results and discussion}

Sodium urate or calcium pyrophosphate dihydrate triclinic and monoclinic crystals were observed in samples from patients with crystal-induced synovi-

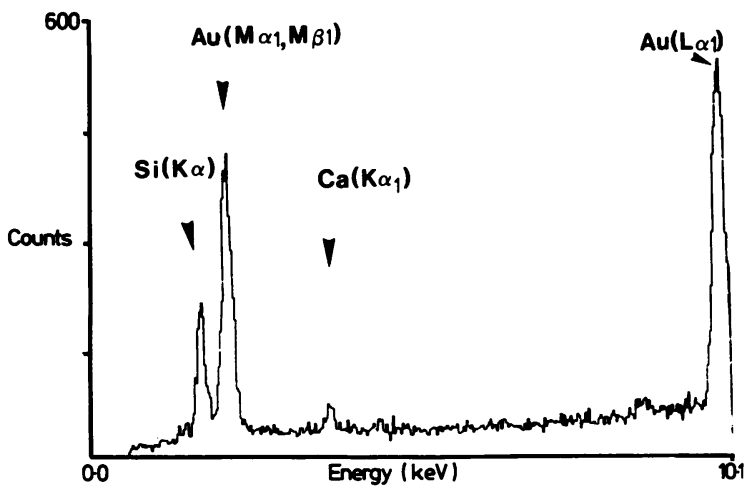

Fig. $2 \mathrm{X}$-ray microanalysis of a particle such as that depicted in Fig. 1, showing the peaks of silicon, calcium, and metallising gold.

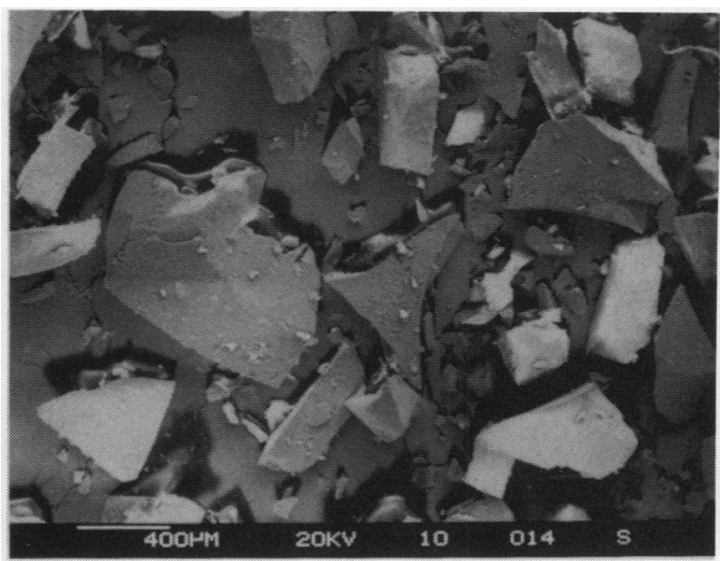

Fig. 3 Glass particles from a ground glass Pasteur pipette, similar to those seen in Fig. $1 .(\times 1000)$.

tis. The composition and morphology of these crystals have been described elsewhere. ${ }^{2} 3$

Frequently a few strange particles were observed, mainly in samples prepared by the second technique (Fig. 1); some were angle shaped, others rod-like, and they usually were larger than natural microcrystals. Systematic microanalysis of these pseudocrystals always showed the presence of silicon, sometimes associated with small amounts of calcium (Fig. 2 ). With experience it was possible to distinguish these silicon crystals by their shape from natural and synthetic triclinic and monoclinic calcium pyrophosphate dihydrate, sodium urate, and apatite conglomerates.

The presence of silicon-containing particles in synovial fluid samples prepared with glass material appeared to be essentially artefactual. Indeed crystallographers seem to consider it an obvious and therefore unreported feature, such as sodium chloride crystals induced by air drying. ${ }^{3}$ Scanning electron microscopy of glass particles from a ground glass Pasteur pipette (Fig. 3) supported the 'technological' origin of such particles in human samples. They can be found even in samples collected in plastic syringes and without glass beads. The recently reported case ${ }^{4}$ of one patient using glass fibres in his work raises the question of a possibly pathogenic role, but caution in the processing of samples has to be kept in mind. Experimental procedures with similar compounds either in vitro or in animal models would be welcome before considering a relationship between this exogeneous material and inflammatory synovitis. 


\section{References}

1 Gaucher A, Faure G, Netter P, Malaman B, Steinmetz J. Identification of microcrystals in synovial fluid by combined scanning electron microscopy and $x$-ray diffraction: application to triclinic calcium pyrophosphate dihydrate. Biomed Express 1977; 27: 242-4.

2 Gaucher A, Faure G, Netter P, Pourel J, Duheille J. Etude des éléments figurés du liquide synovial en microscopie à balayage. Rev Rhum Mal Osteoartic 1979; 46: 249-54.

3 Faure G, Netter P, Malaman B, Steinmetz J, Duheille J,
Gaucher A. Scanning electron microscopic study of microcrys tals implicated in human rheumatic diseases. Scan Electron Microsc 1980; III: 163-76.

4 Cleland L G, Vernon-Roberts B, Smith K. Fibre glass induce synovitis. Ann Rheum Dis 1984; 43: 530-4.

5 Bardin T, Schumacher H R, Lansaman J, Rothfuss S, Dryll A $\overline{\overline{\bar{S}}}$ Transmission electron microscopic identification of silicon-? containing particles in synovial fluid, potential confusion with calcium pyrophosphate dihydrate and apatite crystals. Anno Rheum Dis 1984; 43: 624-7. 